Elder Marino Mendoza Orbegoso

\title{
Estudo Numérico da Radiação Térmica e sua Interação com a Fuligem Formada na Combustão Turbulenta de Combustíveis Líquidos e Gasosos
}

Tese de Doutorado apresentada ao programa de Pós-graduação em Engenharia Mecânica do Departamento de Engenharia Mecânica da PUC-Rio como requisito parcial para obtenção do grau de Doutor em Engenharia Mecânica

Orientador : Prof. Luís Fernando Figueira da Silva Co-Orientador: Dr. Ricardo Serfaty 


\section{Elder Marino Mendoza Orbegoso}

\section{Estudo Numérico da Radiação Térmica e sua Interação com a Fuligem Formada na Combustão Turbulenta de Combustíveis Líquidos e Gasosos}

Tese de Doutorado apresentada ao Programa de Pós-graduação em Engenharia Mecânica como requisito parcial para a obtenção do grau de Doutor em Engenharia Mecânica do Departamento de Engenharia Mecânica do Centro Técnico Científico da PUC-Rio. Aprovada pela Comissão Examinadora abaixo assinada.

Prof. Luís Fernando Figueira da Silva

Orientador

Departamento de Engenharia Mecânica — PUC-Rio

Dr. Ricardo Serfaty

Co-Orientador

Centro de Pesquisas - PETROBRAS

Prof. Andrés Fuentes

Universidad Técnica Federico Santa Maria de Chile - UTFSM

Prof. Amir Antônio Martins de Oliveira Júnior Universidade Federal de Santa Catarina - UFSC

Prof. Guenther Carlos Krieger Filho Universidade de São Paulo - USP

Prof. Angela Ourivio Nieckele

Departamento de Engenharia Mecânica - PUC-Rio

Prof. Marcos Sebastião de Paula Gomes Departamento de Engenharia Mecânica - PUC-Rio

Prof. José Eugênio Leal

Coordenador Setorial do Centro Técnico Científico - PUC-Rio 
Todos os direitos reservados. proibida a reprodução total ou parcial do trabalho sem autorização da universidade, do autor e do orientador.

\section{Elder Marino Mendoza Orbegoso}

Formou-se Engenheiro Mecânico na Universidad Nacional de Trujillo - Peru. Fez mestrado em Engenharia Mecânica na Pontifícia Universidade Católica do Rio de Janeiro Brasil. Atualmente, trabalha como pesquisador no Centro Técnico Científico da Pontifícia Universidade Católica do Rio de Janeiro - Brasil.

Ficha Catalográfica

Mendoza Orbegoso, Elder Marino

Estudo Numérico da Radiação Térmica e sua Interação com a Fuligem Formada na Combustão Turbulenta de Combustíveis Líquidos e Gasosos / Elder Marino Mendoza Orbegoso; orientador: Luís Fernando Figueira da Silva; coorientador: Ricardo Serfaty. - Rio de Janeiro : PUC-Rio, Departamento de Engenharia Mecânica, 2013.

v., 282 f: il. ; $29,7 \mathrm{~cm}$

1. Tese (doutorado) - Pontifícia Universidade Católica do Rio de Janeiro, Departamento de Engenharia Mecânica.

Inclui referências bibliográficas.

1. Engenharia Mecânica - Tese. 2. Dinâmica dos fluidos computacional. 3. Combustão turbulenta não prémisturada. 4. Equação de transferência radiativa. 5. Teoria de Rayleigh. 6. Modelos de fase discreta. I. Figueira da Silva, Luís Fernando. II. Serfaty, Ricardo. III. Pontifícia Universidade Católica do Rio de Janeiro. Departamento de Engenharia Mecânica. IV. Título. 
A quens se encontram me aguardando na vida venidera: meu filho Gabriel Benjamín, meus avós Angel Marino e Floriza Consuelo, e meus tios Melver Marino e Maritza Luzmila. 


\section{Agradecimentos}

A Deus por tudo o acontecido na minha vida.

A PUC-Rio, pela conseção de insenção de pagamento no programa de Doutorado em Engenharia Mecânica.

Ao Departamento de Engenharia Mecânica da PUC-Rio e ao Centro de Pesquisa da Petrobras, pela oportunidade de trabalhar no Projeto Conveniado "Desenvolvimento de Metodologia Baseada em CFD para Otimização de Queimadores Industriais".

A meu orientador, o Professor Luis Fernando, graças a seus conhecimentos e ensinamentos e por ter acreditado em minha pessoa para trabalhar no desenvolvimento de projetos de pesquisa.

A meu Co-orientador Ricardo Serfaty, obrigado pelo suporte e pelas sugestões feitas, como responsável do Projeto Conveniado entre a PUC-Rio e a Petrobras, ao longo destes três anos.

A minha mãe Miriam Ofelia, fonte inesgotável de esforço e sacrifício, obrigado pelo amor, sabedoria e ajuda permanente em todas as etapas da minha vida.

A minha esposa Maica Carolin e meus filhos Andy Samuel, Franco Emiliano e Elder Gustavo; agradeço vocês pelo amor infinito que me concedem e pela muita paciência que vocês tiveram em todo este tempo.

A meu irmão, Oscar Salomón, obrigado pelo amor fraterno e por ser uma pessoa especial que inspira respeito e admiração.

A meus amigos, Américo Barbosa Cunha Jr., Andrea Carvalho dos Anjos, Fernando de Andrade Oliveira, Juan José Cruz Villanueva, Luís Enrique Alva Huapaya, Nattan Roberto Caetano, Paul Ortega Sotomayor, obrigado pelos momentos compartilhados ao longo de minha permanência na PUC-Rio. 


\section{Resumo}

Mendoza Orbegoso, Elder Marino; Figueira da Silva, Luís Fernando; Serfaty, Ricardo. Estudo Numérico da Radiação Térmica e sua Interação com a Fuligem Formada na Combustão Turbulenta de Combustíveis Líquidos e Gasosos. Rio de Janeiro, 2013. 282p. Tese de Doutorado - Departamento de Engenharia Mecânica, Pontifícia Universidade Católica do Rio de Janeiro.

O presente trabalho apresenta um estudo numérico da transferência de energia por radiação e sua interação com as propriedades radiantes cinzas e espectrais dos gases produtos da combustão e da fuligem que são formados em um processo de combustão turbulenta. Assim, utilizam-se sistemas de forno/queimador que operam em regime de chama não pré-misturada de maneira a avaliar, através da dinâmica dos fluidos computacional (CFD), a influência que exercem os diversos modelos de propriedades radiantes sobre a representação da termoquímica do escoamento reativo. Com o objetivo de identificar as principais características e deficiências que apresentam cada um destes modelos, foram considerados dois cenários. O primeiro, correspondente a um problema de radiação unidimensional de um sistema homogêneo e não isotérmico onde são estudados, modelos de propriedades radiantes (i) disponíveis em um software comercial de CFD e (ii) aqueles que foram implementados neste trabalho. Além disso, foi empregado um código numérico que determina as propriedades radiantes espectrais de gases produtos da combustão e da fuligem através de uma abordagem de banda estreita. Para este fim, este código foi acoplado com o software de CFD. Em seguida, dois queimadores de porte laboratorial são empregados de forma a avaliar a capacidade preditiva dos modelos de propriedades radiantes: o primeiro queima propano gasoso e ar enriquecido com oxigênio e o segundo utiliza querosene líquido e oxigênio como reagentes. Dados experimentais de fluxo de calor radiante e de fração volumétrica da fuligem são utilizados para comparação com os resultados obtidos da simulação. Para ambas as configurações de queimador foi também estudado o modelo de Moss-Brookes para previsão da formação/consumo da fuligem. Os resultados obtidos demonstraram o bom desempenho da maioria dos modelos de propriedades radiantes estudados. Em particular, a abordagem de banda estreita foi o que melhor previu a radiação térmica. Além disso, a sua utilização com o modelo de Moss-Brookes levou à melhor previsão da fração volumétrica da fuligem.

\section{Palavras-chave}

Dinâmica dos fluidos computacional; Combustão turbulenta não prémisturada; Equação de transferência radiativa; Teoria de Rayleigh; Modelos de fase discreta; 


\section{Abstract}

Mendoza Orbegoso, Elder Marino; Figueira da Silva, Luís Fernando (Advisor); Serfaty, Ricardo (Co-advisor). Numerical study of the interaction between thermal radiation and soot formation in the turbulent combustion of liquid and gaseous fuels. Rio de Janeiro, 2013. 282p. PhD Thesis - Departamento de Engenharia Mecânica, Pontifícia Universidade Católica do Rio de Janeiro.

This work presents a numerical study of radiation heat transfer and its interaction with gray and spectral radiation of combustion products and soot that are formed in a turbulent combustion process. Different burner/furnace systems operating in a non-premixed combustion regime were used in order to evaluate, through computational fluid dynamics (CFD), the influence of several radiant properties models. Aiming to identify the key features and shortcomings that exhibit each of these models, two scenarios were considered. The first corresponds to a 1-D radiation problem where radiative properties models of a homogeneous non isothermal system are studied as (i) available CFD commercial software and (ii) those implemented in this work. Moreover, a numerical code was used in order to determine, through a narrow band approach, the spectral radiative properties of soot and combustion products. For this purpose, this code was coupled with the CFD software. Then, two laboratory-scale burners are used to assess the predictive capacity of radiative properties models: the first, burning propane and enriched air oxygen, and the second uses kerosene and oxygen as reactants. Measurements of radiant heat flux and soot volumetric fraction are used for comparison with simulation results. For both configurations, the performance of the Moss-Brookes model for predicting the soot production was also studied. The results of this study demonstrated the good performance of the majority of the radiant properties models studied. Particularly, the narrow band approach was the model that provided the best thermal radiation prediction. Moreover, the combination of the narrow band approach with the Moss-Brookes model lead to the best prediction of soot volume fraction.

\section{Keywords}

Computational Fluid Dynamics; Non-Premixed Turbulent Combustion; Radiative Transfer Equation; Rayleigh Theory; Discrete Phase Models; 


\section{Sumário}

1 Introdução $\quad 36$

1.1 Motivação 36

1.2 Delimitação do Problema 41

1.3 Objetivo 43

1.4 Organização do trabalho 44

2 Revisão Bibliográfica $\quad 46$

2.1 Estudos Experimentais de Queimadores Não Pré-Misturados 47

2.2 Estudos Numéricos de Queimadores Não Pré-Misturados 54

2.3 Estudos de Queimadores Desenvolvidos pela International Flame Research Foundation $\quad 68$

2.4 Considerações Finais $\quad 72$

3 Formulação Matemática e Modelos para a Descrição da Combustão Turbulenta em Queimadores

3.1 Equações de Transporte e de Estado que descrevem os Escoamentos Turbulentos Segundo a Abordagem Média de Reynolds 75

3.2 Modelos de Fechamento 77

3.3 Formulação Matemática do Transporte e Evaporação de Gotículas (Droplets) Segundo a Abordagem Euleriana-Lagrangiana

4 Descrição do Radcal e Estudo dos Modelos de Propriedades Radiantes de Gases Produtos da Combustão e da Fuligem

4.1 O Radcal como Ferramenta Complementar na Determinação da Propriedades Radiantes

4.2 Estudo das Propriedades Radiantes dos Produtos da Combustão e da Fuligem

$\begin{array}{ll}\text { 4.3 Considerações Finais } & 164\end{array}$

$5 \quad$ Resultados do Estudo do Queimador de Endrud (2000) 167

5.1 Descrição da Geometria, Malha e Condições de Contorno 168

5.2 Resultados e Discussões 174

6 Resultados do Estudo Numérico do Queimador de "Spray" de Nakamura et al. (2011)

6.1 Geometria e da Malha do Queimador de Nakamura et al. (2011) 217

6.2 Condições de Operação e de Contorno 218

6.3 Resultados e Discussões 221

7 Conclusões e Recomendações $\quad 238$

$\begin{array}{lll}7.1 & \text { Conclusões } & 238\end{array}$

7.2 Recomendações para Trabalhos Futuros 242

$\begin{array}{ll}\text { Referências Bibliográficas } & 244\end{array}$ 
A Cinética Química das Reações de Combustão $\quad 256$

A.1 Estado da Arte 256

A.2 Mecanismos de Cinética Química Detalhada 257

B Dados de Entrada e Saída do Programa Radcal e Interface em UDF para o Acoplamento Radcal/Fluent ${ }^{T M} \quad \mathbf{2 6 6}$

B.1 Parâmetros de Entrada 266

B.2 Parâmetros de Saída 269

B.3 Implementação da Interface em UDF para o Acoplamento entre o Radcal e o Fluent ${ }^{T M}$

C Coeficiente de Absorção Global Mediante o Modelo de Soma Ponderada de Gases Cinzas (WSGGM) - Programação em UDF do modelo WSGGM(Mossi)

C.1 Função Definida Pelo Usuário (UDF) para o modelo WSGGM(Mossi) 273

D Coeficiente de Absorção Espectral da Fuligem Segundo a Teoria de Rayleigh - Formulação e Programação em UDF

D.1 Teoria de Absorção/Espalhamento de Rayleigh para o Cálculo das Propriedades Radiantes da Fuligem

D.2 Cálculo do Coeficiente de Absorção Equivalente da Fuligem Através da Teoria de Rayleigh e Aplicando-se a Metodologia de Discretização Valor Médio

D.3 Funções Definidas Pelo Usuário (UDF)

E Levantamento das Curvas de Vaporização para o n-heptano de ndodecano(querosene) 


\section{Lista de figuras}

1.1 it Esquema simplificado de um sistema de combustão de fornos e caldeiras.

2.1 Diagrama esquemático da bancada experimental e do queimador "twin-fluid" (Fisher e Moss, 1998).

2.2 Perfis de fração de mistura, temperatura, fração volumétrica da fuligem e irradiância obtidos por Fisher e Moss (1998) no queimador de querosene líquido e ar a 2 bar.

2.3 Diagrama esquemático do (a) queimador Elco-Mat RPD60 e, (b) forno de teste VUB (Vrije Universiteit Brussel) de $20 \mathrm{MW}$ (Colson et al., 1999).

2.4 Evolução da temperatura e espécies químicas estáveis obtida por Colson et al. (1999) como função de distância radial, $r / D$, para a posição longitudinal $x / D=0,26$. L. esquerdo: Perfis de $C O_{2}(\square)$, $\mathrm{O}_{2} / 2(\bigcirc), N O x \times 10^{3}(\triangle)$ e $T(\diamond)$. L. direito: Perfis de $C O / 50$ $(\bigcirc), C_{2} H_{2}(\triangle), C_{2} H_{4}(\square), C_{4} / 200(\diamond)$ e $C_{2} H_{6} / 10(+)$.

2.5 Diagrama esquemático, fotografias do funcionamento e dados do queimador de Köhler et. al. (2011).

2.6 Fração volumétrica da fuligem obtidos do queimador de Köhler et. al., (2011). (a) perfil longitudinal no eixo de simetria, (b) perfil radial, (c) campo instantâneo e, (d) campo médio.

2.7 Diagrama esquemático (a) do sistema de fornecimento de reagentes e, (b) dos equipamentos utizilados na experimentação: 1.- tanque de oxigênio, 2.- tanque de Nitrogênio, 3.- tanque de combustível líquido, 4.- medidor de vazão, 5.- queimador 6.- atomizador twinfluid, 7.- câmara de alta pressão, 8.- válvula de alívio, 9.- válvula de controle de pressão, 10.- janela de quarzo, 11.- laser de Argônio, 12.- fibra óptica, 13.- transmissor PDA, 14.- Receptor PDA, 15.Processador de sinais, 16.- osciloscópio, 17.- PC para PDA, 18. PC para travessia e, 19.- trilho óptico. (Nakamura et al., 2011).

2.8 Visualização de chamas em "spray" (superior) e de gotículas de querosene (inferior) para faixas pressões entre 0,1 e $1,0 \mathrm{MPa}$ (Nakamura et al., 2011).

2.9 Distribuição longitudinal de (a) velocidade longitudinal das gotículas de querosene, (b) diâmetro médio Sauter e (c) temperatura média do gas, para pressões de de $0,1,0,3,0,5,0,7 \mathrm{e}$ 1, $0 \mathrm{MPa}$ (Nakamura et al., 2011).

2.10 Ilustração esquemática do injetor protótipo de 200 kW (Spangelo, 2004).

2.11 Contornos da componente longitudinal de velocidade igual a zero obtidos empregando-se os modelos de turbulência (a) $k-\varepsilon$, (b) RNG $k-\varepsilon$, (c) RSM, (Spangelo, 2004). As siglas CRZ e CTRZ significam, respectivamente, à zona de recirculação no canto [Corner Recirculation Zone] e à zona de recirculação toroidal no centro [Central Toroidal Recirculation Zone]. 
2.12 Campos de temperatura obtidos com os modelos de combustão (a) Eddy Dissipation, (b) PDF em equilíbrio, (c) PDF em elemento de chama (Spangelo, 2004).

2.13 Modelo geométrico de uma câmara de combustão com um queimador de dois estágios, e as 27 combinações radial/axial e tangencial dos injetores secundários (Sarlej et al., 2007).

2.14 Campo de temperatura obtido nos caso do arranjo dos bicos injetores secundários que fornecem valores de $N O$ (a) mínimo e (b) máximo (Sarlej et al., 2007).

2.15 Perfis longitudinal e radial da fração volumétrica da fuligem obtidos com modelos de Moss-Brookes e Moss Brookes estendido (Wen et al., 2003).

2.16 Diagrama esquemático do queimador industrial de Wu e Fricker (1976) e resultados de temperatura e fluxo de calor radiante obtidos por Cumber e Fairweather (2005).

2.17 Variação longitudinal e radial do fluxo de calor radiante no queimador laboratorial de Baillie et al. (1998).

2.18 Diagrama esquemático do queimador de Endrud (2000).

2.19 Casos analisados por Wang et al. (2005) no estudo da sensibilidade da fuligem sobre a radiação usando o método dos momentos, e levando em conta os seguintes parâmetros: mecanismo de crescimento de superfície, fator estérico e acoplamento radiação-fuligem.

2.20 Comparação dos resultados de simulação de Wang et al. (2005) com os dados experimentais de Wang et al. (2002) do fluxo de energia radiante ao longo da parede longitudinal do queimador de Endrud (2000).

2.21 Campos de temperatura obtidos mediante a simulação numérica (a) com radiação e (b) sem radiação (Bidi et al., 2008).

2.22 Perfis longitudinais da temperatura e frações mássicas das espécies majoritárias no centro do queimador (Bidi et al., 2008).

2.23 Vista esquemática do forno IFRF (Mahmud e Sangha, 2010).

2.24 Comparação entre os perfis radiais de temperatura preditos por Mahmud e Sangha (2010) e medidos por Michelfelder e Lowes (1974). As linhas tracejadas correspondem ao modelo $k-\varepsilon$ e as linhas contínuas ao modelo tensor de Reynolds (STR).

2.25 Configuração geral do queimador para forno de alumínio fundido e resultados numéricos do fluxo de calor por radiação e total, obtidos com (a) gás natural e (b) óleo combustível (Nieckele et. al., 2011).

2.26 Diagrama esquemático do forno CAGCT e do queimador CGRI (Poirier et. al., 2006).

2.27 Comparação entre os resultados experimentais (esquerda) e numéricos (direita) do processo da combustão turbulenta no plano transversal localizado a $x=215 \mathrm{~mm}$ a jusante do queimador CGRI (Fleck et. al., 2003).

2.28 Lado esquerdo: Diagrama esquemático da câmara de combustão. Lado direito: (a) fotografia da chama, e distribuição da fração de mistura de oxidação obtida por (b) Modelo Elemento de Chama e, (c) Modelo Eddy Break-Up (Weihong e Wlodzimierz, 2006). 
2.29 Forno de Teste HiTAC (a) Lado esquerdo.- Fotografia do equipamento e diagrama esquemático do domínio computacional, (b) Lado direito.- Resultados calculados e medidos de concentração de oxigênio (a $x=0$ e $z=0,6$ ) e de formação de $N O x$ na saida do queimador nas configurações de HCR (Weihong e Wlodzimierz, 2006).

3.1 Diagrama esquemático de chamas não pré-misturada em contrafluxo, mostrando o processo de mistura entre combustível e oxidante (Law, 2006).

3.2 Fotografia típica obtida através de microscópio de transmissão eletrônica que identifica a natureza física da fuligem em chamas de ar/acetileno (Koylu and Faeth 1994).

3.3 Método de ordenadas discretas em um octante de uma esfera unitária (a) $S_{2}$, (b) $S_{4}$ e (c) $S_{6}$.

3.4 Volume de controle usado na representação tridimensional do modelo de ordenadas discretas.

3.5 Interação entre ondas electormagnéticas e partículas esféricas (Modest, 2003).

3.6 Função Fase para os espalhamentos Rayleigh e isotrópico (Siegel, 2002). Neste gráfico $\beta$ corresponte ao ângulo polar $\theta$.

3.7 Comparação do índice de refração determinado por (a) - Chang e Charalampopoulos (1990) (b) - - - Lee e Tien (1980), e, (c) -.-.- Dalzell and Sarofim (1969). [Extraído de Chang e Charalampopoulos (1990)].

4.1 Estrutura do Programa Radcal (Grosshandler, 1993).

4.2 Diagrama esquemático do problema unidimensional de placa plana. 142

4.3 Malha e condições de contorno empregados na simulação.

4.4 Resultado típico de cálculo em CFD da radiação de gases confinados entre placas paralelas e infinitas (a) temperatura do meio, $T$, (b) coeficiente de absorção global, $a$, e (c) radiação incidente total, G. 144

4.5 Influência do número de discretização de ângulos sólidos, $N A S=$ $N_{\phi} \times N_{\theta}$, no divergente do fluxo de calor radiante em placas planas paralelas, $d q_{r} / d y$.

4.6 Divergente do fluxo de calor radiante em placas plana paralelas. Resultados da simulação de radiação de gases produtos da combustão empregando-se os modelos WSGGM(Smith), WSGGM(Mossi), Radcal e Linha por Linha de Mossi (2011).

4.7 Divergente do fluxo de calor radiante obtidos com os modelos (a) Radcal e (b) WSGGM(Smith), para comprimentos médio do feixe de $1,5 L$ e $1,8 L$. O modelo WSGGM(Mossi) para $L_{m}=1,8 L$ é também apresentado para fins de referência.

4.8 Radiação incidente obtidos com os modelos (a) Radcal e (b) WSGGM(Smith), para comprimentos médio do feixe iguais a $1,0 L$, $1,5 L$ e $1,8 L$. O modelo WSGGM(Mossi) para $L_{m}=1,8 L$ é também apresentado para fins de referência. 
4.9 Coeficiente de absorção global obtidos com os modelos (a) Radcal e (b) WSGGM, para comprimentos médio do feixe iguais a $1,0 \mathrm{~L}$, $1,5 L$ e $1,8 L$. O modelo WSGGM(Mossi) para $L_{m}=1,8 L$ é também apresentado para fins de referência.

4.10 Divergente do fluxo de calor radiante de gases produtos da combustão em placa plana paralela empregando-se o modelo Radcal.

4.11 Intensidade de radiação espectral e coeficiente de absorção espectral obtidos do Radcal para uma mistura de gases homogênea e isotérmica empregando-se a abordagem de Modelo Gás Cinza. Coordenadas (a) Natural e (b) Logaritmica.

4.12 Intensidade de radiação espectral e coeficiente de absorção espectral obtidos do Radcal para uma mistura de gases homogênea e isotérmica mediante a abordagem de Modelo de Banda Larga-Cinza (GWB) para $N_{b}=2$ faixas de comprimento de onda (a) $[1-12,5]$ $\mu \mathrm{m}$, (b) $[12,5-200] \mu \mathrm{m}$.

4.13 Divergente do fluxo de calor radiante da mistura de gases produto da combustão e fuligem obtidos com os modelos WSGGM(Smith)\&Sazhin, WSGGM(Smith)\&Rayleigh e Radcal.

4.14 Radiação Incidente da mistura de gases produto da combustão e fuligem obtidos com os modelos WSGGM(Smith)\&Sazhin, WSGGM(Smith)\&Rayleigh e Radcal.

4.15 Coeficiente de absorção global da mistura de gases produto da combustão e fuligem obtidos com os modelos WSGGM(Smith)\&Sazhin, WSGGM(Smith)\&Rayleigh e Radcal.

4.16 Divergente do fluxo de calor radiante da mistura de gases produtos da combustão e fuligem, obtido do modelo WSGGM(Smith)\&Rayleigh, para os casos em que o espalhamento é e não é levado em conta. (a) $Y_{\text {soot }}=0,01$ e (b) $Y_{\text {soot }}=0,001$.

4.17 Radiação incidente da mistura de gases da mistura de gases produtos da combustão e fuligem, obtido com o modelo WSGGM(Smith)\&Rayleigh, para os casos em que o espalhamento é e não é levado em conta. (a) $Y_{\text {soot }}=0,01$ e (b) $Y_{\text {soot }}=0,001$.

4.18 Coeficientes global de absorção e de espalhamento da mistura de gases produtos da combustão e fuligem, obtido com o modelo WSGGM(Smith)\&Rayleigh, para os casos em que o espalhamento é e não é levado em conta. (a) $Y_{\text {soot }}=0,01$ e (b) $Y_{\text {soot }}=0,001$.

5.1 Diagrama esquemático do queimador empregado nas simulações (Wang et al., 2005).

5.2 Domínio computacional desenvolvido nos softwares DesignModeling e Meshing-Grid empregado nas simulações em Fluent (a) Disposição Frontal, (b) Disposição em perpectiva, e, (c) Detalhe da malha na região de entrada do propano ao queimador

5.3 Esquema representativo no cálculo da fração volumêtrica da fuligem equivalente, $F_{V}^{*}$, a partir de resultados de coeficiente de absorção da fuligem, $a_{\text {soot }}$.

5.4 Comparação do campo de temperatura obtidos pelos modelos (a) DOM-WSGGM(Smith) e P1-FSK, e, (b) e DOM\&MBWSGGM(Smith)\&Sazhin e P1-FSK\&MM. 
5.5 Comparação do campo de fração mássica de (a) dióxido de carbono, e (b) vapor de água, obtidos na presente simulação com os modelos DOM\&MB-WSGGM(Smith)\&Sazhin e P1-FSK\&MM.

5.6 Comparação dos resultados numéricos de Wang et al. (2005) ao longo do eixo de simetria, com aqueles obtidos com os modelos DOM-WSGGM(Smith) e DOM\&MB-WSGGM(Smith)\&Sazhin. (a) Temperatura, (b) fração mássica de dióxido de carbono e, (c) fração mássica de vapor de água.

5.7 Comparação do modelo WSGGM(Smith) e do modelo WSGGM(Smith)\&Sazhin com os resultados experimentais e numéricos de Wang et al. (2005) referente ao (a) fluxo de calor radiante na parede do queimador, $q_{r, P}$, e, (b) fração volumétrica equivalente da fuligem, $F_{V}^{*}$.

5.8 Resultado do acoplamento do modelo elementos de chama não adiabático com o conjundo de modelos DOM\&MBWSGGM(Smith)\&Sazhin. Campos de (a) fração volumétrica da fuligem, $f_{V}$, e das frações molares do (b) acetileno, $X_{C 2 H 2}$, e, (c) hidroxila, $X_{O H}$.

5.9 Balanço da taxa de geração de fração mássica de fuligem resultante da Equação (3-58) no modelo de Moss-Brookes (Brookes e Moss, 1999). Campos de (a) fração volumétrica da fuligem, $f_{V}$, e taxas de (b) nucleação, $\left(\frac{d M}{d t}\right)_{\text {inception, }}$ (c) crescimento da superfície, $\left(\frac{d M}{d t}\right)_{\text {surf,growth }}$, (d) oxidação, $\left(\frac{d M}{d t}\right)_{\text {oxidation }}$, e, (e) líquido $\frac{d M}{d t}$, da fuligem.

5.10 Campos de temperaturas do escoamento obtidos com os modelos e para temperaturas da parede iguais a (a) WSGGM(Smith) (c) $T_{P}=300 \mathrm{~K}$, (b) WSGGM(Smith) @ $T_{P}=400$ K, (c) WSGGM(Smith)\&Sazhin @ $T_{P}=300 \mathrm{~K}$, e, (d) WSGGM(Smith)\&Sazhin @ $T_{P}=400 \mathrm{~K}$.

5.11 Radiação incidente, $G$, obtidos com os modelos e para temperaturas da parede iguais a (a) WSGGM(Smith) @ $T_{P}=300 \mathrm{~K}$, (b) WSGGM(Smith) @ $T_{P}=400 \mathrm{~K}$, (c) WSGGM(Smith)\&Sazhin @ $T_{P}=300 \mathrm{~K}$, e, (d) WSGGM(Smith)\&Sazhin @ $T_{P}=400 \mathrm{~K}$.

5.12 Coeficiente de absorção global, $a$, obtidos com os modelos e para temperaturas da parede iguais a (a) WSGGM(Smith) (c) $T_{P}=300 \mathrm{~K}$, (b) WSGGM(Smith) @ $T_{P}=400$ K, (c) WSGGM(Smith)\&Sazhin @ $T_{P}=300 \mathrm{~K}$, e, (d) WSGGM(Smith)\&Sazhin @ $T_{P}=400 \mathrm{~K}$.

5.13 Fração volumétrica da fuligem, $f_{V}$, obtidas com os modelos e para temperaturas da parede iguais a (a) WSGGM(Smith)\&Sazhin @ $T_{P}=300 \mathrm{~K}$, e, (b) WSGGM(Smith)\&Sazhin @ $T_{P}=400 \mathrm{~K}$.

5.14 Fluxo de calor radiante na parede longitudinal do queimador de Endrud (2000) calculado com os modelos, (a) WSGGM(Smith) @ $T_{P}=300$ e $400 \mathrm{~K}$, e (b) WSGGM(Smith)\&Sazhin @ $T_{P}=300 \mathrm{e}$ $400 \mathrm{~K}$.

5.15 Fração volumétrica equivalente da fuligem, $F_{V}^{*}$, do queimador de Endrud (2000) calculado com o modelo WSGGM(Smith)\&Sazhin, e para $T_{P}=300$ e $400 \mathrm{~K}$. 
5.16 Campos de temperatura e radiação incidente obtidos da simulação usando o modelo WSGGM(Smith)\&Sazhin para (a) $T_{P}=300 \mathrm{~K}$, e, (b) $T_{P}=700 \mathrm{~K}$.

5.17 Campos de fração mássica de $\mathrm{CO}_{2}$ e coeficiente de absorção obtidos da simulação usando o modelo WSGGM(Smith)\&Sazhin para (a) $T_{P}=300 \mathrm{~K}$, e, (b) $T_{P}=700 \mathrm{~K}$.

5.18 Fluxo de calor radiante na parede longitudinal do queimador de Endrud (2000) calculado com o modelo WSGGM(Smith)\&Sazhin para $T_{P}=300$ e $700 \mathrm{~K}$.

5.19 Campos de temperaturas do escoamento obtidos com os modelos (a) WSGGM(Smith)\&Sazhin, (b) WSGGM(Smith)\&Rayleigh, e, (c) Radcal.

5.20 Radiação incidente do escoamento obtidos com os modelos (a) WSGGM(Smith)\&Sazhin, (b) WSGGM(Smith)\&Rayleigh, e, (c) Radcal.

5.21 Fração mássica de dióxido de carbono obtidos com os modelos (a) WSGGM(Smith)\&Sazhin, (b) WSGGM(Smith)\&Rayleigh, e, (c) Radcal.

5.22 Fração volumétrica de fuligem obtidos com os modelos (a) WSGGM(Smith)\&Sazhin, (b) WSGGM(Smith)\&Rayleigh, e, (c) Radcal.

5.23 Coeficiente de absorção global obtidos com os modelos (a) WSGGM(Smith)\&Sazhin, (b) WSGGM(Smith)\&Rayleigh, e, (c) Radcal.

5.24 Comparação com resultados experimentais e numéricas de Wang et al. (2005) ao longo do queimador de Endrud (2000) dos resultados obtidos com modelos WSGGM(Smith)\&Sazhin, WSGGM(Smith)\&Rayleigh e Radcal, no que diz respeito (a) ao fluxo de calor radiante, $q_{r, P}$, e (b) à fração volumétrica equivalente da fuligem, $F_{V}^{*}$.

5.25 Campos de temperatura do escoamento, $T$, obtidos com os modelos (a) WSGGM de Smith et al. (1982) (b) WSGGM de Mossi (2011) e (c) Radcal.

5.26 Coeficiente de absorção dos gases produtos da combustão, $a_{g}$, obtido com os modelos (a) WSGGM de Smith et al. (1982) (b) WSGGM de Mossi (2011) e (c) Radcal.

5.27 Razão das pressões parciais, $p_{\mathrm{H} 2 \mathrm{O}} / p_{\mathrm{CO} 2}$ obtidos com o modelo elementos de chama não adiabático acoplado com os modelos ordenadas discretas e (a) WSGGM de Smith et al. (1982) (b) WSGGM de Mossi (2011) e (c) Radcal.

5.28 Radiação incidente, $G$, obtida com os modelos (a) WSGGM de Smith et al. (1982) (b) WSGGM de Mossi (2011) e (c) Radcal.

5.29 Fração mássica de vapór de água, $Y_{H 2 O}$, obtida com os modelos (a) WSGGM de Smith et al. (1982) (b) WSGGM de Mossi (2011) e (c) Radcal. 
5.30 Fluxo de calor radiante ao longo da parede longitudinal do queimador, $q_{r, P}$, calculados com os modelos WSGGM(Smith), WSGGM(Mossi) e Radcal. Comparação com dados experimentais de Wang et al. (2005).

5.31 Influência do parâmetro de escalonamento de oxidação, $C_{\text {oxid }}$, do modelo de Moss-Brookes. Campos de fração volumétrica de fuligem obtidos com os modelos (a) WSGGM(Smith)\&Sazhin e (b) Radcal. 211

5.32 Influência do parâmetro de escalonamento de oxidação, $C_{\text {oxid }}$, do modelo de Moss-Brookes. Campos de coeficiente de absorção global obtidos com os modelos (a) WSGGM(Smith)\&Sazhin e (b) Radcal. 211

5.33 Influência do parâmetro de escalonamento de oxidação, $C_{\text {oxid }}$, do modelo de Moss-Brookes. Campos de radiação incidente obtidos com os modelos (a) WSGGM(Smith)\&Sazhin, e, (b) Radcal.

5.34 Influência do parâmetro de escalonamento de oxidação, $C_{\text {oxid }}$, do modelo de Moss-Brookes. Campos de temperatura obtidos com os modelos (a) WSGGM(Smith)\&Sazhin, e, (b) Radcal.

5.35 Influência do parâmetro de escalonamento de oxidação, $C_{\text {oxid }}$, do modelo de Moss-Brookes. Campos de fração mássica do dióxido de carbono obtidos da simulação com os modelos (a) WSGGM(Smith)\&Sazhin, e, (b) Radcal.

5.36 Influência do parâmetro de escalonamento de oxidação, $C_{\text {oxid }}$, do modelo de Moss-Brookes. (a) Fluxo de calor radiante, $q_{r, P}$, e (b) fração volumétrica equivalente da fuligem, $F_{V}^{*}$.

6.1 Diagrama esquemático do queimador (a) sistema de fornecimentos e(b) bancada experimental PDA (Nakamura et al., 2011).

6.2 Domínio computacional do queimador de Nakamura et al. (2011) empregado nas simulações. (a) Vista global, (b) Detalhe da malha na região de entrada dos reagentes.

6.3 Fração mássica da fuligem e temperatura obtidos da simulação do queimador de Nakamura et al. (2011) a 0,1 MPa. O campo de temperatura foi comparado com imagens de chama.

6.4 Fração mássica da fuligem e temperatura obtidos da simulação do queimador de Nakamura et al. (2011) a 0,3 MPa. O campo de temperatura foi comparado com imagens de chama.

6.5 Campos de componente longitudinal de velocidade, temperatura e pressão obtidos para o queimador de Nakamura et al. (2011) para $0,1 \mathrm{MPa}$.

6.6 Campos de componente longitudinal de velocidade, temperatura e pressão obtidos pela simulação do queimador de Nakamura et al. (2011) para 0, $3 \mathrm{MPa}$.

6.7 Distribuição radial de temperatura para $z$ iguais a (a) $66,9 \mathrm{~mm}$ e (b) $111,5 \mathrm{~mm}$, obtidos do experimento de Nakamura et al. (2011) e da simulação numérica para $0,1 \mathrm{MPa}$. .

6.8 Distribuição radial de temperatura para $z$ iguais a (a) $15,45 \mathrm{~mm}$ e (b) $51,5 \mathrm{~mm}$, obtidos do experimento de Nakamura et al. (2011) e da simulação numérica. Para $0,3 \mathrm{MPa}$ 
6.9 Operação do queimador a 0,1 MPa. (a) Fração mássica da fuligem calculada e distribuição radial da fração volumétrica da fuligem obtida para (b) $66,9 \mathrm{~mm}$ e (c) $111,5 \mathrm{~mm}$.

6.10 Operação do queimador a 0,3 MPa. (a) Fração mássica da fuligem calculada e distribuição radial da fração volumétrica da fuligem obtida para (b) $30,9 \mathrm{~mm}$ e (c) $51,5 \mathrm{~mm}$.

6.11 Operação do queimador a $0,1 \mathrm{MPa}$. (a) Campo simulado de radiação global incidente, $G$, com o modelo WSGGM(Smith)\&Sazhin, e (b) Comparação qualitativa ao longo da parede, entre a radiação global incidente, $G$, obtida da simulação, e a radiação espectral incidente, $G_{\lambda}$, obtida nos experimentos de Nakamura et al. (2011).

6.12 Operação do queimador a $0,3 \mathrm{MPa}$ a) Campo calculado de radiação global incidente, $G$, com 0 modelo WSGGM(Smith)\&Sazhin, e (b) Comparação qualitativa ao longo da parede, entre a radiação global incidente, $G$, obtido da simulação, e a radiação espectral incidente, $G_{\lambda}$, obtida nos experimentos de Nakamura et al. (2011).

6.13 Operação do queimador a $0,1 \mathrm{MPa}$. Visualização das gotículas obtidos, (a) dos experimentos de Nakamura et al. (2011) e (b) da simulação numérica.

6.14 Operação do queimador a $0,3 \mathrm{MPa}$. Visualização das gotículas obtidos, (a) dos experimentos de Nakamura et al. (2011) e (b) da simulação numérica.

6.15 Distribuição do diâmetro gota calculada para (a) $z=10 \mathrm{~mm}$, e (b) $z=50 \mathrm{~mm}$, na operação do queimador a $0,1 \mathrm{MPa}$.

6.16 Distribuição do diâmetro de gota para (a) $z=10 \mathrm{~mm}$, e (b) $z=$ $50 \mathrm{~mm}$, obtidos dos resultados experimentais de Nakamura et al. (2008), na operação do queimador a $0,1 \mathrm{MPa}$.

6.17 Comparação entre os resultados de simulação e os dados experimentais de Nakamura et al. (2008) para 0,1 MPa: (a) velocidade longitudinal e (b) diâmetro médio das gotículas de combustível líquido.

6.18 Comparação entre os resultados de simulação e os dados experimentais de Nakamura et al. (2008) para 0,3 MPa: (a) velocidade longitudinal e (b) diâmetro médio das gotículas de combustível líquido.

B.1 Discretização considerada pelo programa Radcal para: (a) sistemas não homogêneos e (b) sistemas homogêneos e isotérmicos.

B.2 Dados de saída do RCOUT.dat fornecido pela simulação do Radcal para as condições iniciais descrito pela Tabela A.1 (Grosshandler, 1993).

B.3 Transmitância espectral de um sistema de $0,5 \mathrm{~m}$ de diâmetro a 1 atm e $320 \mathrm{~K}$, para uma mistura de gases composto por $\mathrm{CO}_{2}, \mathrm{H}_{2} \mathrm{O}$, $\mathrm{CH}_{4}, \mathrm{CO}$, e fuligem (Grosshandler, 1993). 
D.1 Curva do coeficiente de absorção espectral da fuligem segundo a teoria de espalhamento Rayleigh e seu equivalente aplicando a abordagem de Modelo de Banda Larga-Cinza (GWB).

D.2 Comparação entre os valores analíticos e de discretização de $C_{\lambda}(\lambda)$ e $K(\lambda)=C_{\lambda}(\lambda) / \lambda$ como função do comprimento de onda.

E.1 Curvas de pressão de saturação do n-heptano e do n-dodecano. 281 


\section{Lista de tabelas}

3.1 Quadraturas $S_{N}$ sobre um octante (Jamaluddin e Smith, 1988). 101

3.2 Coeficientes polinomiais de Smith et al. (1982) utilizados no modelo WSGGM.

3.3 Coeficientes polinomiais de Mossi (2011) utilizados no modelo WSGGM.

3.4 Formulações aplicadas segundo o tipo de partícula e processo de transferência de massa e calor.

4.1 Bandas Moleculares Incluidas no Radcal (Grosshandler, 1993).

4.2 Tempo de processamento como função do número ordenadas discretas $N A S=N_{\phi} \times N_{\theta}$.

4.3 Tempo de processamento obtido com os modelos WSGGM(Smith), WSGGM(Mossi) e Radcal.

4.4 Descrição dos casos simulados para o estudo da influência da separação das faixas de comprimento de onda e do tipo de coeficiente de absorção.

4.5 Resultado da simulação em Radcal aplcando-se as abordagens de Modelo de Gás Cinza (GGM) e Modelo de Banda Larga-Cinza (GWB) para uma problema de radiação unidimensional de uma mistura homogênea e isotérmica.

4.6 Tempo de processamento associado aos modelos WSGGM(Smith)\&Sazhin, WSGGM(Smith)\&Rayleigh e Radcal.

5.1 Condições de contorno utilizada na simulação do queimador de Endrud (2000).

5.2 Constantes do modelo Moss-Brookes empregados para simulação do queimador de Endrud (2000).

5.3 Balanço térmico nos contornos obtido com modelos e para temperaturas da parede iguais a (a) WSGGM(Smith) @ $T_{P}=300 \mathrm{~K}$, (b) WSGGM(Smith) @ $T_{P}=400 \mathrm{~K}$, (c) WSGGM(Smith)\&Sazhin @ $T_{P}=300 \mathrm{~K}$, e, (d) WSGGM(Smith)\&Sazhin @ $T_{P}=400 \mathrm{~K}$.

5.4 Taxa de transferência de calor por radiação nos contornos obtidos com modelos e para temperaturas da parede iguais a (a) WSGGM(Smith) @ $T_{P}=300 \mathrm{~K}$, (b) WSGGM(Smith) @ $T_{P}=$ $400 \mathrm{~K}$, (c) DWSGGM(Smith)\&Sazhin @ $T_{P}=300 \mathrm{~K}$, e, (d) WSGGM(Smith)\&Sazhin @ $T_{P}=400 \mathrm{~K}$.

5.5 Propriedades médias na saída do queimador Endrud (2000) fornecidos pelos modelos e para temperaturas da parede iguais a (a) WSGGM(Smith) @ $T_{P}=300 \mathrm{~K}$, (b) WSGGM(Smith) @ $T_{P}=$ $400 \mathrm{~K}$, (c) WSGGM(Smith)\&Sazhin @ $T_{P}=300 \mathrm{~K}$, e, (d) WSGGM(Smith)\&Sazhin @ $T_{P}=400 \mathrm{~K}$.

5.6 Balanço térmico na parede longitudinal do queimador obtido com o modelo WSGGM(Smith)\&Sazhin, e para temperaturas da parede, $T_{P}$, de (a) $300 \mathrm{~K}$, e, (b) $700 \mathrm{~K}$. Estes resultados são comparado com aquele do experimento de Wang et al. (2002) 
5.7 Taxas de transferência de energia sensível na parede longitudinal e temperatura média na saída do Queimador de Endrud (2000) obtidos com os modelos (a) WSGGM(Smith)\&Sazhin, (b) WSGGM(Smith)\&Rayleigh, e, (c) Radcal.

5.8 Propriedades máximas do escoamento obtidos com os modelos (a) WSGGM(Smith)\&Sazhin, (b) WSGGM(Smith)\&Rayleigh, e, (c) Radcal.

6.1 Condições de contorno utilizadas nas simulações do queimador de Nakamura et al (2011).

6.2 Constantes do modelo Moos-Brookes empregados para simulação do queimador de Nakamura et al. (2011).

B.1 Dados de entrada típicos do arquivo RC.dat para o caso de um sistema isotérmico e homogêneo (Grosshandler, 1993).

B.2 Dados de entrada típicos do arquivo RC.dat para o caso de um sistema não-isotérmico (Grosshandler, 1993).

D.1 Discretização "valor médio". Valores de $C_{o, j}$ e $K_{j}$ aplicados à UDF para cada faixa de comprimento de onda.

E.1 Parâmetros empíricos da Equação de Antoine para o n-heptano e o n-dodecano obtidos do trabalho de Williamham et al. (1945).

E.2 Temperaturas de ebulição do n-heptano e do n-dodecano para 0,1 e 0,3 MPa, obtidas das formulações de Sazhin (2006) e dos dados reportados pelo NIST [Williamham et al. (1945)]. 


\title{
Nomenclatura
}

\author{
Caracteres Latinos
}

a Raio de uma partícula ou gotícula esférica $(\mu \mathrm{m})$.

$a$ Coeficiente de absorção cinza do meio participante $(1 / \mathrm{m})$.

$a_{g} \quad$ Coeficiente de absorção cinza do gás como meio participante $(1 / \mathrm{m})$

$a_{\text {soot }}$ Coeficiente de absorção cinza da fuligem como meio participante $(1 / \mathrm{m})$.

$a_{\epsilon, i} \quad$ Fator peso da emissividade o $i$-ésimo gás cinza.

$a_{E} \quad$ Coeficiente de absorção/emissão médio efetivo $(1 / \mathrm{m})$.

$a_{I} \quad$ Coeficiente de absorção média incidente $(1 / \mathrm{m})$.

$a_{P} \quad$ Coeficiente de emissão média de Planck $(1 / \mathrm{m})$.

$a_{\text {Geom }}$ Coeficiente médio geométrico $(1 / \mathrm{m})$.

$a_{\text {Patch }}$ Coeficiente de Patch $(1 / \mathrm{m})$.

$a_{\lambda} \quad$ Coeficiente de absorção espectral do meio participante $(1 / \mathrm{m})$.

$a_{\lambda, g} \quad$ Coeficiente de absorção espectral do gas $(1 / \mathrm{m})$.

$a_{\lambda, \text { soot }}$ Coeficiente de absorção espectral da fuligem $(1 / \mathrm{m})$.

$\vec{a}_{p} \quad$ Vetor aceleração de uma gotícula $\left(\mathrm{m} / \mathrm{s}^{2}\right)$.

$A \quad$ Área de uma superficie $\left(\mathrm{m}^{2}\right)$.

$A_{o}, A_{s} \quad$ Coeficientes relativos ao modelo k- $\varepsilon$ realizável.

$A_{k} \quad$ Fator pre-exponencial da $k$-ésima reação elementar direta.

$A_{p} \quad$ Área da seção transversal de uma gotícula $\left(\mathrm{m}^{2}\right)$.

$\widetilde{b}_{n u c}^{*} \quad$ Conc. dos núcleos radicais de fuligem normalizada a $10^{15}$ $\mathrm{mol} / \mathrm{part}$.

$b_{1}, b_{T} \quad$ Constantes do modelo de Sazhin.

$b_{\epsilon, i j} \quad j$-ésimo coeficiente polinomial do $i$-ésimo gás cinza.

c Distância entre partículas $(\mu \mathrm{m})$. 
c Velocidade da luz $(\mathrm{m} / \mathrm{s})$.

$c_{p} \quad$ Calor específico a pressão constante do fluido $(\mathrm{J} / \mathrm{kg})$.

$c_{p, p} \quad$ Calor específico a pressão constante da gotícula $(\mathrm{J} / \mathrm{kg})$.

$c_{p, \infty} \quad$ Calor específico a pressão constante da fase contínua $(\mathrm{J} / \mathrm{kg})$.

C Coeficiente da função fase.

$C_{1}, C_{2}$ Coeficientes relativos ao modelo k- $\varepsilon$ realizável.

$C_{s}, C_{t}$ Constantes de Talbot.

$C_{D} \quad$ Coeficiente de arrasto.

$C_{D, e s f}$ Coeficiente de arrasto de gotículas de forma esférica.

$C_{i, s} \quad$ Concentração de vapor da $i$-ésima espécie na superfície da gotícula $\left(\mathrm{kmol} / \mathrm{m}^{3}\right)$.

$C_{i, \infty} \quad$ Concentração de vapor da $i$-ésima espécie no meio contínuo $\left(\mathrm{kmol} / \mathrm{m}^{3}\right)$.

$C_{L} \quad$ Constante do modelo $\mathrm{k}-\varepsilon$ segundo a abordagem de rastreio estocástico.

$C_{a b s} \quad$ Seção transversal de absorção $\left(\mathrm{m}^{2}\right)$.

$C_{\text {ext }}$ Seção transversal de extinção $\left(\mathrm{m}^{2}\right)$.

$C_{\text {sca }}$ Seção transversal de espalhamento $\left(\mathrm{m}^{2}\right)$.

$C_{\text {oxid }}$ Fator de escala da taxa de oxidação da fuligem.

$C_{\alpha} \quad$ Constante associada à taxa de nucleação da fuligem.

$C_{\beta} \quad$ Constante associada à taxa de coagulação da fuligem.

$C_{\gamma} \quad$ Fator de escala do crescimento da superfície da fuligem.

$C_{\mu} \quad$ Fator empírico na relação Prandtl-Kolmogorov.

$C_{\chi} \quad$ Constante do modelo elemento de chama [Flamelet].

$C_{\omega} \quad$ Constante do consumo da fração mássica da fuligem devido à oxidação.

$d \quad$ Diâmetro interno do injetor $(\mathrm{m})$.

$d_{p} \quad$ Diâmetro de uma partícula ou gotícula (m).

D Diâmetro interno do queimador $(\mathrm{m})$. 
D Coeficiente de difusão molecular das espécies químicas $\left(\mathrm{m}^{2} / \mathrm{s}\right)$.

$D_{i, m} \quad$ Coeficiente de difusão do vapor no volume $[b u l k]\left(\mathrm{m}^{2} / \mathrm{s}\right)$.

$D_{T, p} \quad$ Coeficiente de termoforese.

$E_{k} \quad$ Energia de ativação da $k$-ésima reação elementar direta.

$f_{v, 0} \quad$ Fração de compostos voláteis na gotícula.

$f_{V} \quad$ Fração volumétrica da fuligem.

$F_{V}^{*} \quad$ Fração volumétrica equivalente da fuligem.

$F_{D} \quad$ Frequência de arrasto $(1 / \mathrm{s})$.

$\vec{F} \quad$ Vetor forças adicionais (N).

$\vec{F}_{t f} \quad$ Força de termoforese (N).

$g_{i} \quad$ Aceleração da gravidade na direção do $i$-ésimo componente de $\vec{x}\left(\mathrm{~m} / \mathrm{s}^{2}\right)$.

$\vec{g} \quad$ Vetor aceleração da gravidade $\left(\mathrm{m} / \mathrm{s}^{2}\right)$.

G Radiação incidente total $\left(\mathrm{W} / \mathrm{m}^{2}\right)$.

$G_{\lambda} \quad$ Radiação incidente espectral $\left(\mathrm{W} / \mathrm{m}^{2} \mu \mathrm{m}\right)$.

$h \quad$ Coeficiente de transferência de calor por convecção $\left(\mathrm{W} / \mathrm{m}^{2} \mathrm{~K}\right)$.

$h_{a d} \quad$ Entalpia total em sistema adiabático $(\mathrm{J})$.

$h_{f g, p} \quad$ Entalpia [calor latente] de vaporização da gotícula $(\mathrm{J})$.

$h_{F} \quad$ Entalpia do combustível (J).

$h_{O} \quad$ Entalpia do oxidante $(\mathrm{J})$.

$h_{t} \quad$ Entalpia total $(\mathrm{J})$.

$i_{1}, i_{2} \quad$ Intensidades polarizadas.

I Intensidade de radiação total $\left(\mathrm{W} / \mathrm{m}^{2} \mathrm{sr}\right)$.

$I_{b} \quad$ Intensidade de radiação total emitida por um corpo negro $\left(\mathrm{W} / \mathrm{m}^{2} \mathrm{sr}\right)$.

$I_{\lambda} \quad$ Intensidade de radiação espectral $\left(\mathrm{W} / \mathrm{m}^{2} \mathrm{sr} \mu \mathrm{m}\right)$.

$I_{b, \lambda} \quad$ Intensidade de radiação espectral que emite um corpo negro $\left(\mathrm{W} / \mathrm{m}^{2} \mathrm{sr} \mu \mathrm{m}\right)$. 
$I_{\lambda, P} \quad$ Intensidade de radiação espectral da parede isotérmica $\left(\mathrm{W} / \mathrm{m}^{2} \mathrm{sr} \mu \mathrm{m}\right)$.

$\mathcal{I}_{t} \quad$ Intensidade turbulenta.

$k_{c} \quad$ Coeficiente de transferência de massa $(\mathrm{m} / \mathrm{s})$.

$k \quad$ Condutividade térmica do fluido $(\mathrm{W} / \mathrm{m} \mathrm{K})$.

$k_{p} \quad$ Condutividade térmica da gotícula como elemento discreto $(\mathrm{W} / \mathrm{m} \mathrm{K})$.

$k_{\infty} \quad$ Condutividade térmica da fase contínua $(\mathrm{W} / \mathrm{m} \mathrm{K})$.

$k_{k}^{f}, k_{k}^{b} \quad$ Coeficientes direto e reverso de velocidade da $k$-ésima reação.

$K_{n} \quad$ Número de Knudsen.

$K e_{k} \quad$ Constante de equilíbrio da $k$-ésima reação elementar.

$\widetilde{k} \quad$ Energia cinética turbulenta $\left(\mathrm{m}^{2} / \mathrm{s}^{2}\right)$.

$l \quad$ Expoente de nucleação no modelo Moss-Brookes.

$l_{t} \quad$ Comprimento integral característico da turbulência.

$L \quad$ Distância de separação entre as placas planas $(\mathrm{m})$.

$L_{m} \quad$ Comprimento médio do feixe $(\mathrm{m})$.

$\mathcal{L} \quad$ Escala de comprimento integral do turbilhão $(\mathrm{m})$.

$m \quad$ Expoente da taxa de crescimento de superfície no modelo MossBrookes.

$m$ Índice complexo de refração.

$m_{k} \quad$ Expoente da temperatura da $k$-ésima reação elementar direta.

$m_{p} \quad$ Massa atual de uma gotícula $(\mathrm{kg})$.

$m_{p, 0} \quad$ Massa inicial de uma gotícula $(\mathrm{kg})$.

$\dot{m}_{F} \quad$ Vazão mássica de combustível.

$\dot{m}_{O x} \quad$ Vazão mássica de oxidante.

$M_{P} \quad$ Massa molecular de uma partícula incipiente de fuligem $(\mathrm{kg} / \mathrm{kmol})$.

$\bar{M}_{\text {soot }}$ Concentração mássica média da fuligem $\left(\mathrm{kg} / \mathrm{m}^{3}\right)$.

$\overline{M W} \quad$ Peso molecular de uma mistura de fase contínua $(\mathrm{kg} / \mathrm{kmol})$. 
$\overline{M W}_{i} \quad$ Peso molecular $i$-ésima espécie química $(\mathrm{kg} / \mathrm{kmol})$.

$\overline{M W}_{p} \quad$ Peso molecular da gotícula $(\mathrm{kg} / \mathrm{kmol})$.

Ma Número de Mach.

$\mathcal{M}_{i} \quad$ Símbolo químico da espécies $i$.

$n \quad$ Parte real do indice de refração.

n Expoente da taxa de crescimento de superfície no modelo Moss-

Brookes.

$\vec{n} \quad$ Vetor unitário normal à uma superficie.

$N \quad$ Número de espécies químicas.

$N_{A} \quad$ Número de Avogadro [6, $\left.022045 \times 10^{23} \mathrm{part} / \mathrm{mol}\right]$.

$N_{g} \quad$ Número de gases cinzas no modelo WSGGM.

$N_{\text {norm }}$ Fator de conversão, igual a $10^{15} \mathrm{~mol} / \mathrm{part}$ de fuligem.

$\bar{N}_{n u c} \quad$ Concentração média das partículas de fuligem dos núcleos radicais $\left(\right.$ part $\left./ \mathrm{m}^{3}\right)$.

$N_{p} \quad$ Número de gotículas dentro de um volume de controle.

$\dot{N}_{i} \quad$ Fluxo molar de vapor da $i$-ésima espécie $\left(\mathrm{kmol} / \mathrm{m}^{2} \mathrm{~s}\right)$.

Nu Número de Nusselt.

$p \quad$ Pressão $(\mathrm{Pa})$.

$\bar{p} \quad$ Pressão média $(\mathrm{Pa})$.

$p_{\text {sat }} \quad$ Pressão de vapor saturado da gotícula $(\mathrm{Pa})$.

Pr Número de Prandtl.

$\mathcal{P} \quad$ Produção da energia cinética turbulenta.

$q \quad$ Parte imaginaria do indice de refração.

$q_{r} \quad$ Fluxo total de calor por radiação normal à superfície $\left(\mathrm{W} / \mathrm{m}^{2}\right)$.

$\dot{q}_{k} \quad$ Taxa de progresso da $k$-ésima reação elementar.

$\dot{q}_{i k} \quad$ Taxa de conversão da $i$-ésima espécie na $k$-ésima reação elementar.

$\vec{q}_{r} \quad$ Vetor fluxo total de calor por radiação $\left(\mathrm{W} / \mathrm{m}^{2}\right)$.

$Q_{a b s} \quad$ Fator de eficiência referente à absorção. 
$Q_{\text {ext }} \quad$ Fator de eficiência referente à extinção.

$Q_{\text {sca }} \quad$ Fator de eficiência referente ao espalhamento.

$Q(\vec{x}, t)$ Propriedade qualquer do escoamento.

$\overline{Q(\vec{x}, t)}$ Média temportal da propriedade $Q(\vec{x}, t)$.

$Q^{\prime}(\vec{x}, t)$ Flutuação temportal da propriedade $Q(\vec{x}, t)$.

$\dot{Q}_{r, P} \quad$ Taxa de transferência de calor por radiação transferida à parede $(\mathrm{W})$.

$\dot{Q}_{T, P} \quad$ Taxa de transferência de calor total transferida à parede $(\mathrm{W})$.

$\vec{r} \quad$ Vetor posição $(\mathrm{m})$.

$\vec{r}_{P} \quad$ Vetor posição da parede $(\mathrm{m})$.

$\mathcal{R}_{0} \quad$ Parâmetro associado ao coeficiente de espalhamento.

$R \quad$ Constante universal dos gases perfeitos $(\mathrm{kJ} / \mathrm{kmol} \mathrm{K})$.

$\overline{\mathcal{R}}_{\text {soot }}$ Taxa de produção/geração de fuligem.

$\overline{\mathcal{R}}_{n u c}^{*} \quad$ Taxa de produção/geração dos núcleos radicais de fuligem.

$R e_{d} \quad$ Número de Reynolds baseado no diâmetro e velocidade relativa de uma gotícula.

$R e_{t} \quad$ Número de Reynolds turbulento.

$s \quad$ Razão entre frações mássicas oxidante/combustível na estequiometria.

$s \quad$ Caminho óptico $(\mathrm{m})$.

$\vec{s} \quad$ Vetor unitário na direção do espalhamento (m).

$\overrightarrow{s^{\prime}} \quad$ Vetor unitário na direção da radiação incidente $(\mathrm{m})$.

S Módulo do tensor taxa de deformação média.

$S_{1}, S_{2} \quad$ Funções de amplitude complexa.

$\widetilde{S}_{i j} \quad$ Tensor da taxa de deformação média.

Sc Número de Schmidt.

Sh Número de Sherwood.

$\bar{S}_{1} \quad$ Termo fonte da equação de conservação da massa resultante da interação interfásica. 
$\bar{S}_{h_{t}} \quad$ Termo fonte da equação de conservação de energia resultante da interação interfásica.

$\bar{S}_{r} \quad$ Termo fonte da equação de conservação de energia como resultado da radiação térmica.

$\bar{S}_{u i} \quad$ i-ésimo termo fonte da equação de conservação de quantidade de movimento produto da interação interfásica.

$\bar{S}_{Z} \quad$ Termo fonte da equação de conservação da fração de mistura média.

$\bar{S}_{Z^{\prime \prime 2}} \quad$ Termo fonte da equação de conservação da variância da fração de mistura.

$t \quad$ Tempo $(s)$.

$t_{0} \quad$ Tempo inicial (s).

T Temperatura instantânea do fluido (K).

$T_{b p} \quad$ Temperatura de ebulição do líquido (K).

$T_{p} \quad$ Temperatura de uma gotícula $(\mathrm{K})$.

$T_{P} \quad$ Temperatura de contorno [parede] $(\mathrm{K})$.

$T_{\text {vap }}$ Temperatura de evaporação do líquido (K).

$T_{\alpha} \quad$ Temperatura de ativação da nucleação da fuligem (K).

$T_{\gamma} \quad$ Temperatura de ativação do crescimento da fuligem (K).

$T_{\infty} \quad$ Temperatura local da fase contínua $(\mathrm{K})$.

$\widetilde{T} \quad$ Temperatura média do fluido $(\mathrm{K})$.

$\widetilde{T}_{\text {saida }}$ Temperatura média do fluido na saída do queimador $(\mathrm{K})$.

$\mathcal{T}_{L} \quad$ Escala de tempo integral lagrangeana do fluido (s).

$\mathcal{T}_{\vec{u}_{p}} \quad$ Escala de tempo integral da gotícula (s).

$u_{i} \quad i$-ésima componente do vetor velocidade $\vec{u}(\mathrm{~m} / \mathrm{s})$.

$u_{p, i} \quad i$-ésima componente da velocidade de uma gotícula $\vec{u}_{p}(\mathrm{~m} / \mathrm{s})$.

$\vec{u} \quad$ Vetor velocidade $(\mathrm{m} / \mathrm{s})$.

$\vec{u}_{p} \quad$ Vetor velocidade de uma gotícula $(\mathrm{m} / \mathrm{s})$.

$U^{*} \quad$ Parâmetro do modelo k- $\varepsilon$ realizável. 
$w_{i} \quad$ Pesos de quadratura associados às direções $\vec{s}_{i}$.

$\mathcal{W}_{\lambda} \quad$ Espessura óptica espectral.

$\vec{x} \quad$ Vetor posição $(\mathrm{m})$.

$x_{i} \quad i$-ézima componente do vetor posição $\vec{x}$.

$X_{i} \quad$ Fração molar da $i$-ésima especie química.

$\left[X_{i}\right] \quad$ Concentração [molar] da $i$-ésima espécie química $\left(\mathrm{kmol} / \mathrm{m}^{3}\right)$.

$\left[X_{\text {soot }}\right]$ Concentração mássica da fuligem $\left(\mathrm{kg} / \mathrm{m}^{3}\right)$.

$X_{F} \quad$ Fração molar de combustível.

$X_{O} \quad$ Fração molar de oxigênio.

$\widetilde{X}_{\text {prec }}$ Fração molar média da espécie precursora na formação da fuligem.

$\widetilde{X}_{\text {sgs }} \quad$ Fração molar média das espécies precursoras na crescimento da fuligem.

$\widetilde{X}_{O H} \quad$ Fração molar média do radical hidroxila.

$Y_{i} \quad$ Fração mássica da $i$-ésima espécie química.

$Y_{F, 1} \quad$ Fração mássica de combustível no lado de fornecimento de combustível.

$Y_{\mathrm{O}_{2}, 2} \quad$ Fração mássica de oxigênio no lado de fornecimento de oxidante.

$\widetilde{Y}_{\text {soot }} \quad$ Fração mássica média da fuligem.

$\mathcal{Y}_{M} \quad$ Termo de dissipação por dilatação.

Z Fração de mistura.

$Z_{s t} \quad$ Fração de mistura estequiométrica. 


\section{Caracteres Gregos}

$\alpha \quad$ Parâmetro de tamanho.

$\beta \quad$ Coeficiente de extinção global $(1 / \mathrm{m})$.

$\gamma \quad$ Coeficiente de expansão adiabática.

$\Delta s \quad$ Distância que percorre um feixe dentro de $\Delta V(\mathrm{~m})$.

$\Delta t_{p} \quad$ Passo de tempo de integração do meio disperso (s).

$\Delta V \quad$ Volume de controle $\left(\mathrm{m}^{3}\right)$.

$\epsilon_{p} \quad$ Emissividade cinza de uma gotícula.

$\epsilon_{P} \quad$ Emissividade cinza da parede.

$\epsilon_{T} \quad$ Emissividade total do gás no modelo WSGGM.

$\varepsilon \quad$ Taxa de dissipação viscosa $\left(\mathrm{m}^{2} / \mathrm{s}^{3}\right)$.

$\widetilde{\varepsilon} \quad$ Taxa de dissipação da energia cinética turbulenta $\left(\mathrm{m}^{2} / \mathrm{s}^{3}\right)$.

$\eta \quad$ Coeficiente do modelo k- $\varepsilon$ realizável.

$\eta_{c o l} \quad$ Eficiência de colisão no modelo de Moss-Brookes.

$\theta \quad$ Ângulo polar.

$\theta_{R} \quad$ Temperatura de radiação $(\mathrm{K})$.

$\kappa_{i} \quad$ Coeficiente de absorção dependente da pressão (1/atm m).

$\lambda$ Coeficiente de transferência de calor por condução $(\mathrm{W} / \mathrm{m} \mathrm{K})$.

$\lambda \quad$ Comprimento de onda $(\mu \mathrm{m})$.

$\mu \quad$ Primeiro momento estatístico [média] de $\chi_{s t}$.

$\mu \quad$ Viscosidade dinâmica do fluido (Pa.s).

$\mu_{t} \quad$ Viscosidade turbulenta (Pa.s).

$\nu \quad$ Viscosidade cinemática do fluido $\left(\mathrm{m}^{2} / \mathrm{s}\right)$.

$\nu_{t} \quad$ Viscosidade cinemática turbulenta $\left(\mathrm{m}^{2} / \mathrm{s}\right)$.

$\nu_{i k}^{\prime}, \nu_{i k}^{\prime \prime}$ Coeficientes estequiométricos das espécies químicas, $\mathcal{M}_{i}$.

$\xi \quad$ Defeito de entalpia $(\mathrm{J})$.

$\rho_{P} \quad$ Refletividade cinza da parede.

$\rho \quad$ Densidade instantânea do fluido $\left(\mathrm{kg} / \mathrm{m}^{3}\right)$.

$\bar{\rho} \quad$ Densidade média do fluido $\left(\mathrm{kg} / \mathrm{m}^{3}\right)$. 
$\rho_{p} \quad$ Densidade de uma gotícula $\left(\mathrm{kg} / \mathrm{m}^{3}\right)$.

$\rho_{\text {soot }}$ Densidade de uma partícula incipiente de fuligem $\left(\mathrm{kg} / \mathrm{m}^{3}\right)$.

$\sigma \quad$ Constante de Stefan-Boltzman [igual a $5,67 \times 10^{8} \mathrm{~W} / \mathrm{m}^{2} \mathrm{~K}^{4}$ ].

$\sigma^{2} \quad$ Segundo momento estatístico [variância] de $\chi_{s t}$.

$\sigma_{Y} \quad$ Número de Schmidt turbulento.

$\sigma_{h} \quad$ Número de Prandtl turbulento.

$\sigma_{k} \quad$ Número de Prandtl turbulento relativo à energia cinética turbulenta.

$\sigma_{n u c} \quad$ Número de Prandtl turbulento relativo ao número de densidade da fuligem.

$\sigma_{s} \quad$ Coeficiente de espalhamento cinza $(1 / \mathrm{m})$.

$\sigma_{s, \lambda} \quad$ Coeficiente de espalhamento espectral $(1 / \mathrm{m})$.

$\sigma_{\text {soot }} \quad$ Número de Prandtl turbulento relativo à fração mássica da fuligem.

$\sigma_{Z} \quad$ Número de Schmidt turbulento relativo à fração de mistura média.

$\sigma_{Z^{\prime \prime 2}} \quad$ Número de Schmidt turbulento relativo à variância da fração de mistura.

$\sigma_{\varepsilon} \quad$ Número de Prandtl turbulento relativo à taxa de dissipação turbulenta.

$\tau \quad$ Variável que relaciona ao tempo (s).

$\tau_{\text {cros }} \quad$ Tempo de travessia de uma gotícula (s).

$\tau_{e} \quad$ Tempo de vida característico de um turbilhão (s).

$\tau_{p} \quad$ Tempo de arrasto de uma gotícula (s).

$\tau_{\lambda} \quad$ Transmitância espectral.

$\phi \quad$ Riqueza de combustível na mistura.

$\chi \quad$ Taxa de dissipação instantânea do campo escalar (1/s).

$\chi_{s t} \quad$ Taxa de dissipação instantânea do campo escalar na estequiometria $(1 / \mathrm{s})$. 
$\omega \quad$ Espalhamento albedo.

$\dot{\omega}_{i} \quad$ Taxa de produção da $i$-ésima espécie química.

$\overline{\dot{\omega}}_{i} \quad$ Média temporal da taxa de reação da i-ézima espécie química.

$\omega_{k} \quad$ Vetor de rotação angular.

$\Omega^{\prime} \quad$ Ângulo sólido.

$\widetilde{\Omega}_{i j} \quad$ Tensor taxa de rotação média.

$\bar{\Omega}_{i j} \quad$ Tensor taxa de rotação média visto desde um referencial rotativo. 
Simbologia de Operadores e Funções

$B(a, b) \quad$ Função Beta de valores complexos $a$ e $b,(\Re\{a\}, \Re\{b\}>0)$.

$e_{i j k} \quad$ Símbolo de permutação.

exp Função exponencial.

$\int_{a}^{b} f(x) d x$ Integral definida de $f(x)$ e de limites $a \leq x \leq b$.

$\lim f(x) \quad$ Limite de uma função $f(x)$.

ln $\quad$ Função logaritmo natural.

max $\quad$ Função máximo.

$\partial / \partial x \quad$ Operador derivada parcial em $x$.

$N\left(d_{p}\right) \quad$ Distribuição de diâmetros de partículas de fuligem.

$P(Q) \quad$ Função Densidade de Probabilidade da variável de amostragem $\mathcal{Q}$.

$\bar{Q} \quad$ Média temporal de Reynolds da propriedade $Q$.

$Q^{\prime} \quad$ Flutuação temporal da prop. $Q$ em torno da média de Reynolds.

$\widetilde{Q} \quad$ Média temporal de Favre da propriedade $Q$.

$Q^{\prime \prime} \quad$ Flutuação temporal da prop. $Q$ em torno da média de Favre.

$\widetilde{Q^{\prime \prime 2}} \quad$ Variância da prop. $Q$ em torno da média de Favre.

$\delta \quad$ Função delta de Dirac.

$\delta_{i j} \quad$ Delta de Kronecker.

$\Phi \quad$ Função fase de espalhamento.

$\nabla . \quad$ Operador divergente.

$\nabla^{2} \quad$ Operador Laplaciano.

$\Im\{z\} \quad$ Parte imaginâria de um número complexo $z$.

$\Re\{z\} \quad$ Parte real de um número complexo $z$.

$\sum \quad$ Operador Somatória.

$\prod \quad$ Operador Produtório. 
Abreviações

BM Modelos de banda.

CRZ Zona de recirculação no canto.

CTRZ Zona de recirculação toroidal no centro.

DNS Simulação numérica direta.

DPM Modelos de fase discreta.

DTRM Modelo de radiação de transferência discreta.

EDM Modelo dissipação dos turbilhões.

EBU Modelo de quebra dos turbilhões.

FRC Modelo taxa química finita.

FTIR Espetroscopia infravermelha de transformada de Fourier.

g-RTE Equação de transferência [transporte] radiante cinza.

GBM Abordagem de banda cinza.

GGM Abordagem de gás cinza.

GWB Abordagem de banda larga-cinza.

HACA Abstração de hidrogênio e adição de acetileno.

LES Simulação de grandes escalas.

LII Incandescência induzida por laser.

LII-2D Incandescência induzida por laser bidimensional.

LBL Abordagem linha por linha.

MM Método dos momentos.

NAS Número de ângulos sólidos.

NBM Abordagem de banda estreita.

PAH Hidrocarbonetos aromáticos policíclicos.

PDA Anemometria de fase Doppler.

PIV Velocimetria por imagens de partículas.

PLIF Incandescência induzida por laser plano.

RANS Média de Reynolds da equação de Navier-Stokes.

RNG Grupo de renormalização. 
RSM Modelo de tensões de Reynolds.

RTE Equação de transferência [transporte] de energia radiante.

s-RTE Equação de transferência radiante sob a forma espectral.

SNB Modelo de banda estreita estatística.

SNBCK Modelo de banda estreita estatística correlacionada no comprimento de onda.

SVCARS Espectrometria anti-Stokes coerente de deslocamento vibracional.

TTNH Modelo não Homogêneo de transmitância total.

UDF Função definido pelo usuário.

WBM Abordagem de banda larga.

WSGGM Soma ponderada dos gases cinzas. 
Lo único que se yergue entre un hombre y lo que desea de la vida, a menudo simplemente es la voluntad de intentarlo y la fe de creer que es posible.

Richard M. Devos, La Universidad del Éxito de Og Mandino. 